\title{
Clinical, hematological, and cytogenetic profile of adult myelodysplastic syndrome in a tertiary
} \section{care center}

This article was published in the following Dove Press journal:

Journal of Blood Medicine

23 February 2017

Number of times this article has been viewed

\section{Santhosh Narayanan}

Department of Medicine, Government Medical College, Kozhikode, Kerala, India
Correspondence: Santhosh Narayanan Department of Medicine, Government Medical College, 27/685 Kottooli, Kozhikode 673016, Kerala, India Tel +9l 9895658833

Email drsanthosh4@gmail.com
Background: Myelodysplastic syndrome (MDS), a disorder of clonal hematopoiesis, is an important clinical entity, but most of the studies available are conducted among the Western population. Its etiological factors and clinicohematological profile in the Indian population are quite diverse. The information regarding its prognostic factors and cytogenetics is very scarce. Objectives: (1) To assess the clinicohematological profile, cytogenetics, prognostic factors, and outcome of MDS and (2) to study its progression to acute myeloid leukemia (AML) in the selected patients over the study period.

Methods: A prospective observational study was performed with patients from Department of Medicine and Hematology, Government Medical College, Kozhikode, who were diagnosed with MDS within the study period (from 1 January 2014 to 31 July 2015). Secondary causes of dysplasia were excluded. In possible cases, the international prognostic scoring system was followed. These patients were followed up for an additional 6 months to assess the progression of MDS to AML based on symptoms, signs, hemogram, or repeat peripheral smear/bone marrow studies.

Results: Of the 60 patients, $73 \%$ were aged $>60$ years. Disease was common in males, with a male:female ratio of 7:3. Thirty-five percent of the patients were working in agricultural and allied fields and had pesticide exposure. Patients with prior radiation exposure had significant association with adverse outcome. Fatigue was the prominent symptom and was reported by $90 \%$ of the patients. Blasts were $>5 \%$ in peripheral smear; bone marrow cytopenia and dysplasia at the time of diagnosis had significant association with risk of transforming to AML. Refractory anemia (RA), observed in 22 patients, was the most common type of MDS. Most of the patients with RA with excess blasts type-1 and RA with excess blasts type-2 transformed to AML, and the association was statistically significant. Deletion of short arm of fifth chromosome ( $5 \mathrm{q}$ deletion) was detected in 11 patients. All of them showed good response to treatment with lenalidomide and had a favorable outcome.

Conclusion: This study highlights the various etiological factors, and the clinical profile of MDS seen in the Indian population. Cytogenetic analysis and application of the international prognostic scoring system has a significant bearing on the outcome, as exemplified by the response to lenalidomide in patients with $5 \mathrm{q}$ deletion. This study also indicates that proper diagnostic and prognostic assessment is necessary to institute appropriate therapeutic options. Keywords: MDS, cytogenetics, AML, prognosis

\section{Introduction}

Myelodysplastic syndromes (MDSs) are a group of heterogeneous hematological disorders characterized by cellular and dysmorphic marrow with consequent ineffective blood cell production. Clinical variability of MDS, diversity of histopathological 
phenotypes, and cytogenetic heterogeneity make the diagnosis challenging to the physician. The majority of the primary adult patients with MDS present with features of bone marrow failure. In about one-third of the patients, there is leukemic transformation. MDS is often difficult to recognize as the symptoms can closely resemble those of other diseases. Differential diagnosis includes all secondary causes of bone marrow failure, namely aplastic anemia, paroxysmal nocturnal hemoglobinuria, infections such as tuberculosis, HIV, leishmaniasis, brucellosis, parvovirus infections, and viral hepatitis, autoimmune diseases such as systemic lupus erythematosus, B12 deficiency, and effect due to various drugs and toxins. Due to the nonspecific nature of histopathological changes, the role of cytogenetics is gaining importance. Clonal cytogenetic abnormalities are found in $30-50 \%$ of de novo cases and $80 \%$ of therapy-related MDS cases. ${ }^{1}$ There is a paucity of data on Indian patients with MDS, with regard to their clinical presentation and their pathological and cytogenetic profiles. Therefore, this study attempts to bring out the clinicopathological and cytogenetic spectrum in the Indian population.

\section{Materials and methods}

This is a prospective observational study of patients who were newly diagnosed with MDS and presenting to the Department of Medicine, Government Medical College, Kozhikode, Kerala, India. The study was commenced on 1 January 2014 and completed on 1 July 2015. Patients were included in the study till 31 December 2014. The selected patients were followed up for additional 6 months (till 1 July 2015) to assess the transformation of MDS to acute myeloid leukemia (AML). All patients $>12$ years of age were included in the study if they met the following criteria of MDS:

1. Unexplained quantitative changes in one or more of the blood and bone marrow elements (ie, red blood cells, granulocytes, or platelets). Following values are used to define cytopenia: hemoglobin, $<10 \mathrm{~g} / \mathrm{dL}(100 \mathrm{~g} / \mathrm{L})$; absolute neutrophil count, $<1.8 \times 10^{9} / \mathrm{L}(<1800 / \mu \mathrm{L})$, and platelets, $<150 \times 10^{9} / \mathrm{L}(<150,000 / \mu \mathrm{L})$. However, failure to meet the threshold for cytopenia does not exclude the diagnosis of MDS if there is definite morphological evidence of dysplasia.

2. Morphological evidence of significant dysplasia (ie, $\geq 10 \%$ of erythroid precursors, granulocytes, or megakaryocytes) upon visual inspection of the peripheral blood smear, bone marrow aspirate, and bone marrow biopsy in the absence of other causes of dysplasia). Sixty patients were included in this study. After obtaining their informed consent, clinical history was collected, and physical examination was performed. The primary investigations undertaken for the diagnosis of MDS include complete hemogram, peripheral smear, bone marrow aspiration, imprint, and trephine biopsy. Cytogenetic profile was determined in possible cases by karyotyping or fluorescence in situ hybridization. Other investigations such as vitamin B12, hepatitis B surface antigen, anti-hepatitis $\mathrm{C}$ virus, HIV by enzyme-linked immunosorbent assay (ELISA), parvovirus by polymerase chain reaction, and immunoglobulin $M$ by ELISA, ANA, anti-dsDNA, and rheumatoid arthritis serology tests were performed when there was a clinical suspicion to rule out secondary causes of dysplasia.

\section{Ethics committee approval and informed consent}

Institutional Ethics Committee of the Government Medical College, Kozhikode, Kerala, approved the research project (ref no. GMCKKD/RP 2014/IEC/16/01). Written informed consent was obtained from all patients who had participated in this study. For patients who were not of legal age to sign on their own behalf, written informed consent was obtained from their guardians.

\section{Results}

A total of 60 patients were included in this study. Of them, $73 \%$ were $>60$ years of age. Most of the patients were in the age group of 70-79 years $(n=22)$ (Figure 1). Among the 60 patients, 42 were males. The male:female ratio was $7: 3$ (Figure 2).

Majority of the male patients were in the age group of $70-79$ years $(n=18)$, whereas most of the female patients were in the age group of $60-69$ years $(n=7)$. Of the total 60 patients,
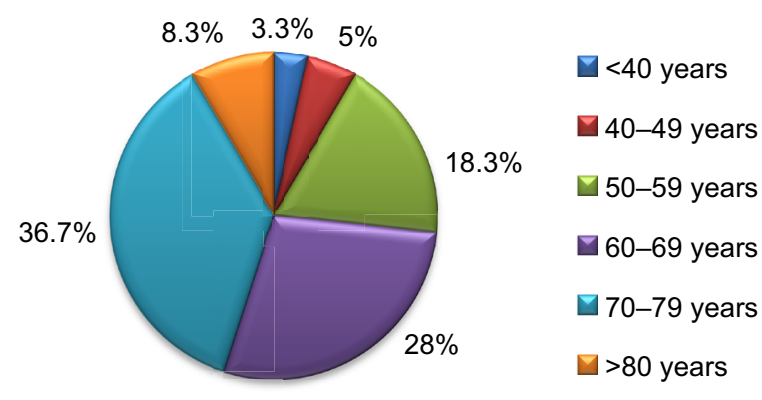

Figure I Age distribution of patients. 


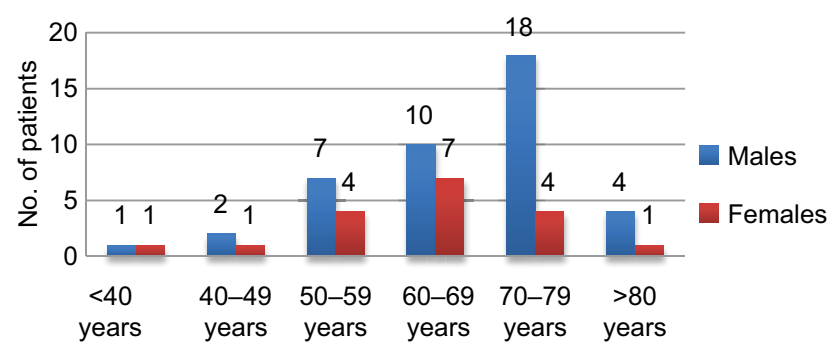

Figure 2 Gender distribution of patients.

$90 \%(\mathrm{n}=54)$ had fatigue, $66.7 \%(\mathrm{n}=40)$ had dyspnea on exertion, 53.3\% $(\mathrm{n}=32)$ had palpitation, $31.7 \%(\mathrm{n}=19)$ had fever, and $33.3 \%(n=20)$ had bleeding. Furthermore, three patients had bone pain (Figure 3).

Of the 60 patients, $35 \%(n=21)$ were working in agricultural and allied fields, $10 \%(\mathrm{n}=5)$ were working in textile industries, and $16 \%(\mathrm{n}=10)$ were having occupation concerned with machineries such as construction work and earthmoving trucks.

Of the 60 patients, seven had prior history of malignancy, and four had history of lymphoma, of which three had nonHodgkin's lymphoma and one had Hodgkin's lymphoma. Two patients had history of breast cancer, and one had germ cell tumor. Two patients had history of treatment with etoposide. Five patients had exposure to cyclophosphamide, and four patients to anthracyclines. Two patients had history of chronic intake of nonsteroidal anti-inflammatory drug for nonspecific musculoskeletal complaints, and one had history of indigenous medications.

Most common physical symptom was pallor as seen in $75 \%$ of the patients $(n=45)$, pedal edema in $36.7 \%(n=22)$, cutaneous bleeding in $28.3 \%(\mathrm{n}=17)$, and retinal hemorrhages

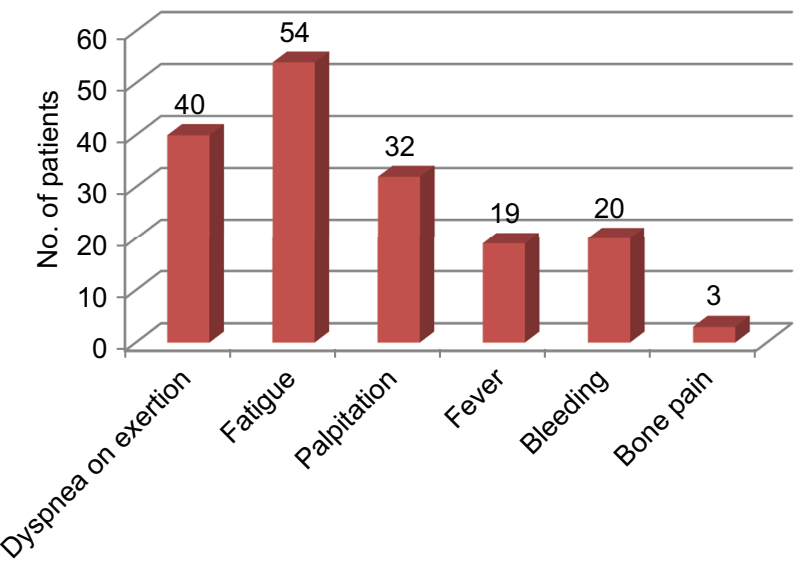

Figure 3 Distribution of symptoms. in $25 \%(\mathrm{n}=15)$. Lymphadenopathy was observed in six patients, and hepatosplenomegaly in four patients (Figure 4).

Of the 60 patients, $33 \%$ had hemoglobin concentration of $<4 \mathrm{~g} / \mathrm{dL}, 42 \%$ had between 4 and $7 \mathrm{~g} / \mathrm{dL}, 15 \%$ had between 7 and $10 \mathrm{~g} / \mathrm{dL}$, and $10 \%$ had $>10 \mathrm{~g} / \mathrm{dL}$. Mean hemoglobin concentration was $5.5 \mathrm{~g} / \mathrm{dL}$ (standard deviation \pm 1.8 ). Of the 60 patients, $28 \%$ had platelet count $<20000 / \mathrm{cmm}, 16 \%$ had it in the range of $20000-50000 / \mathrm{cmm}, 18 \%$ had between 50000 and $1 \mathrm{lakh} / \mathrm{cmm}$, and 13 patients $(21.6 \%) \mathrm{had}>1.5 \mathrm{lakh} / \mathrm{cmm}$, of which seven patients also had chromosome $5 \mathrm{q}$ deletion. Five percent had mean corpuscular volume $<60 \mathrm{fL}, 5 \%$ had it between 60 and $80 \mathrm{fL}, 45 \%$ had it in the range of $100-120 \mathrm{fL}$, and $33 \%$ had it $>120 \mathrm{fL}$.

Most of the patients had macrocytic morphology of RBCs. Blasts in peripheral smear were detected in 11 patients, and 10 patients had pancytopenia. Dimorphic picture was observed in four patients, and nine patients had microcytic RBC morphology (Figure 5).

Cytopenia was observed in $>52 \%$ of the patients $(n=32)$, and dysplasia was observed in 31 patients (51.6). Blasts were present in 22 patients. Ringed sideroblasts was an uncommon finding that was found only in six patients (Figure 6).

Cytogenetic analysis was performed in 52 out of 60 patients. Of them, 11 had deletion of $5 q$ chromosome, whereas four had deletion of $7 \mathrm{q}$ chromosome. Three patients had loss of Y chromosome, whereas 34 patients had normal karyotype (Figure 7).

Figure 8 shows the profile of MDS based on hematological and cytogenetic features.

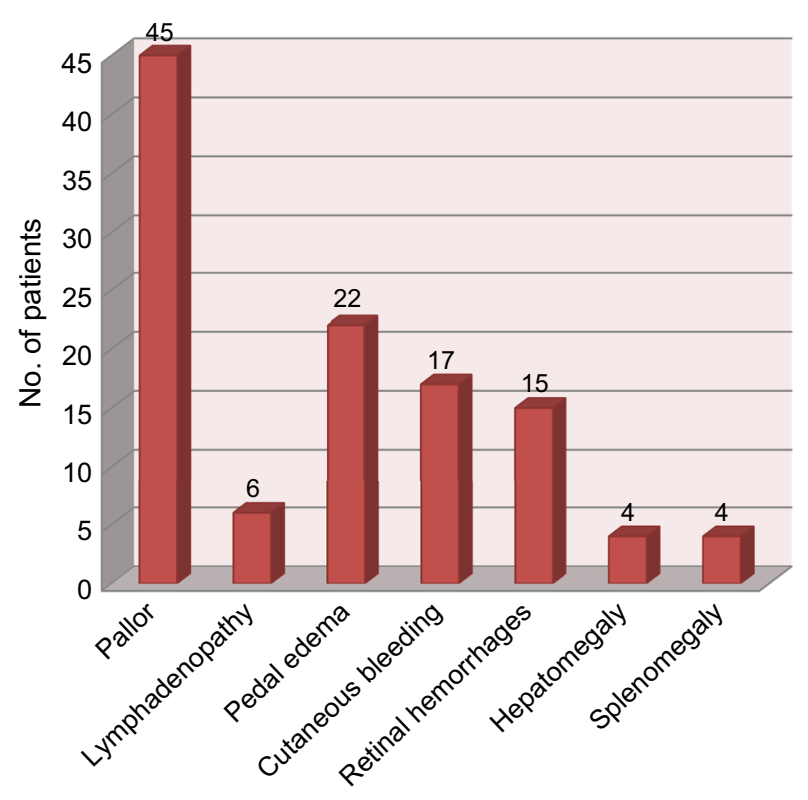

Figure 4 Physical signs. 


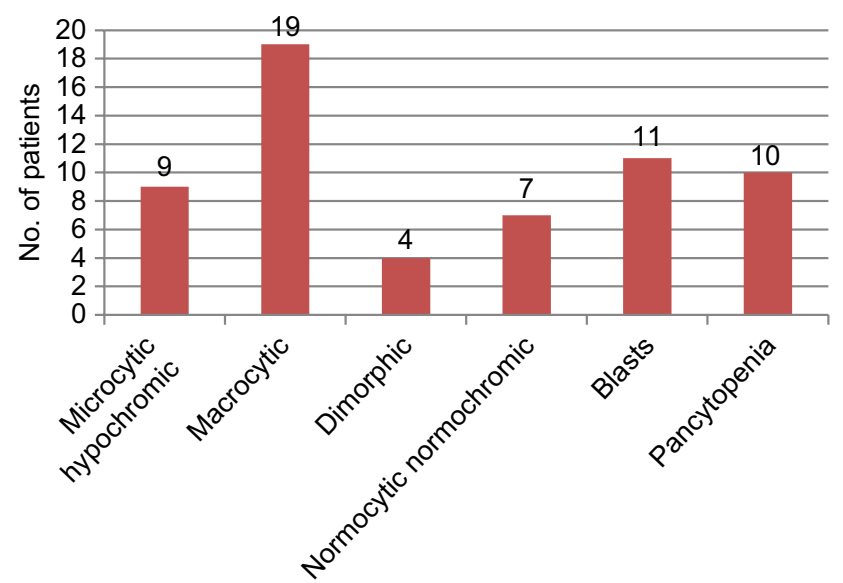

Figure 5 Peripheral smear morphology.

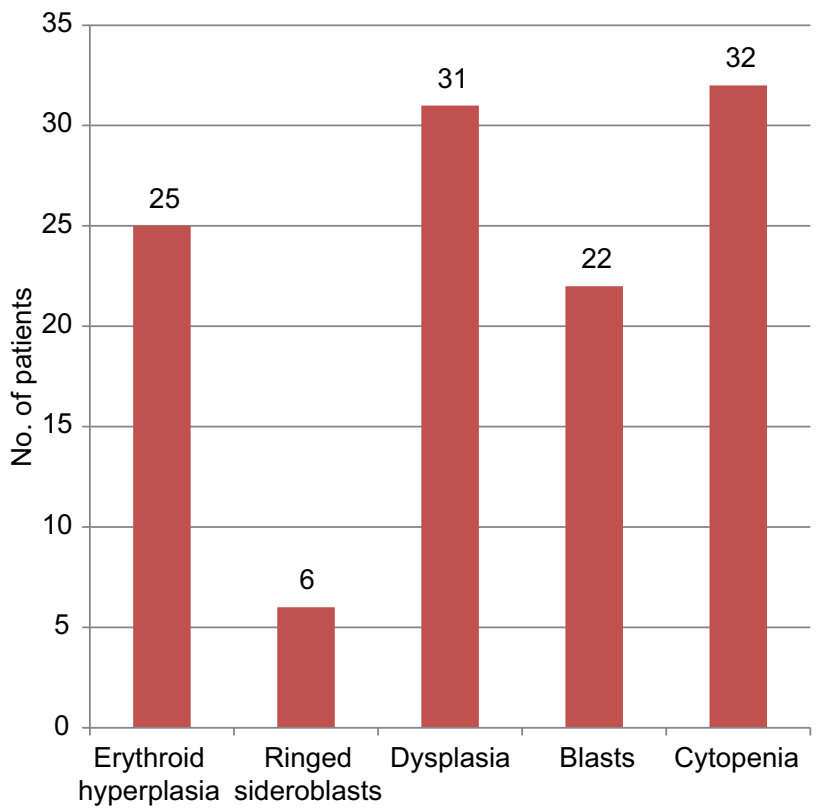

Figure 6 Bone marrow profile.

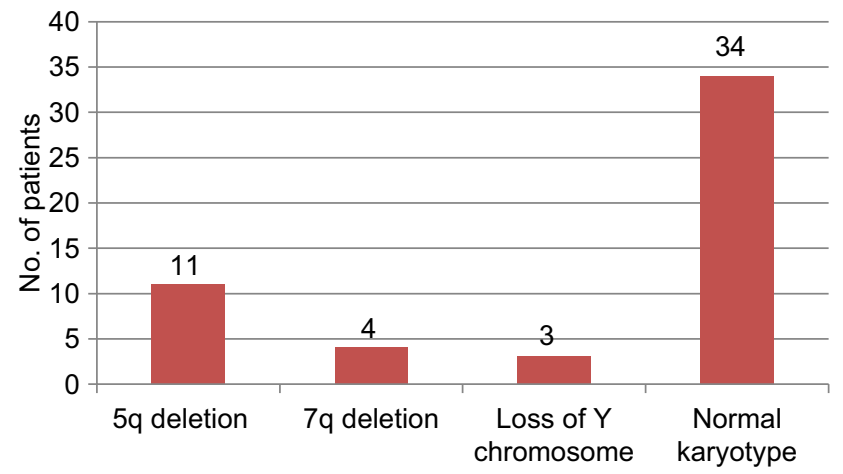

Figure 7 Cytogenetic profile.

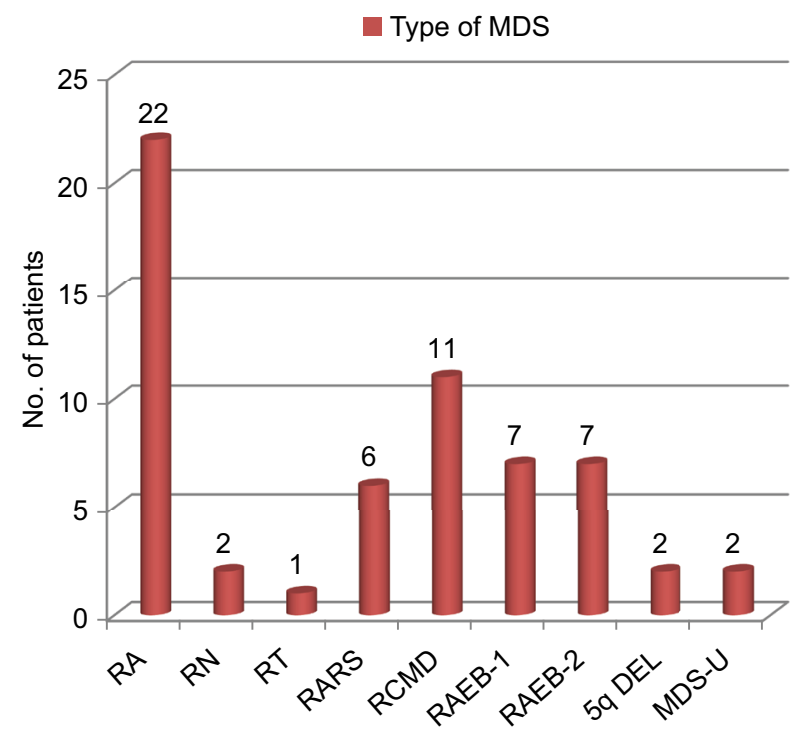

Figure 8 Type of myelodysplastic syndrome.

Abbreviations: MDS, myelodysplastic syndrome; RA, refractory anemia; RN, refractory neutropenia; RT, refractory thrombocytopenia; RARS, refractory anemia with ringed sideroblasts; RCMD, refractory cytopenia with multilineage dysplasia; RAEB-I, refractory anemia with excess blasts type-I; RAEB-2, refractory anemia with excess blasts type-2; $5 q$ del, 5q deletion; MDS, myelodysplastic syndrome; MDS-U, MDS unclassified.

International prognostic scoring system (IPSS) and revised international prognostic scoring system (R-IPSS) were applied for 52 patients who underwent cytogenetic analysis. Based on IPSS stratification, 15 patients had intermediate-2 risk $(\mathrm{n}=15)$, whereas 14 patients had low risk (Figure 9).

Based on R-IPSS stratification, five patients had very low risk, 18 patients had low risk, and 19 patients had intermediate risk of progressing to AML (Figure 10).

Out of 60 patients, 11 patients (10\%) showed MDS-AML transformation. Most common type of MDS-AML transformation was AML-M1 (four patients), followed by AML-M2 and AML-M0 (two patients each) (Figure 11).

It was found that the initial blast percentage in the peripheral smear (Figure 12) had a significant association with future transformation to AML ( $p$-value $=0.000$ ). Significant association was found between blasts $>5 \%$ in bone marrow and those who had cytopenia and dysplasia in bone marrow with a risk of transformation to AML. There was significant association between refractory anemia (RA) with excess blasts type-1, RA with excess blasts type-2, and refractory cytopenia with multilineage dysplasia (RCMD) and risk of transformation to AML ( $p$-value $<0.001$ ). No significant association was found between RA, RA with ringed sideroblasts (RARS), 5q del, and MDS-unclassified and risk of transformation to AML. 


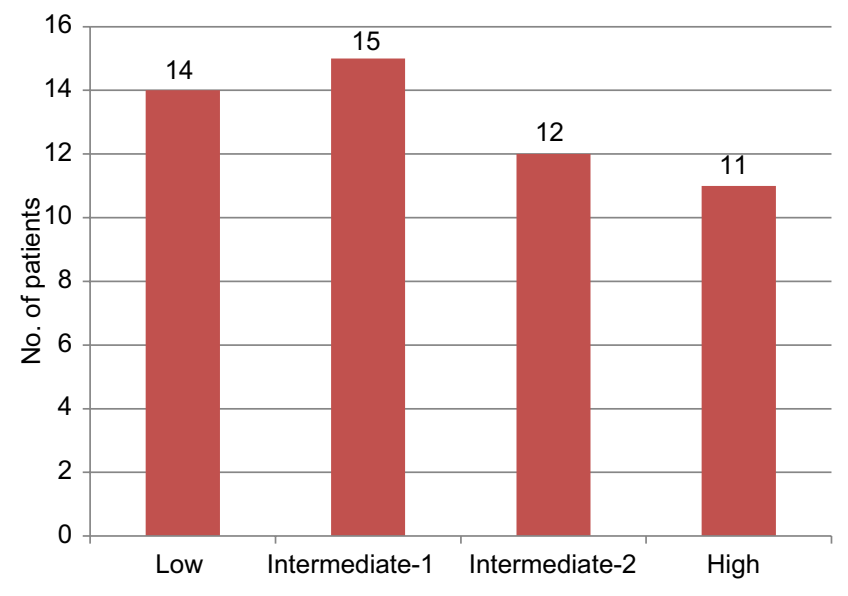

Figure 9 IPSS stratification of patients.

Abbreviations: IPSS, international prognostic scoring system.

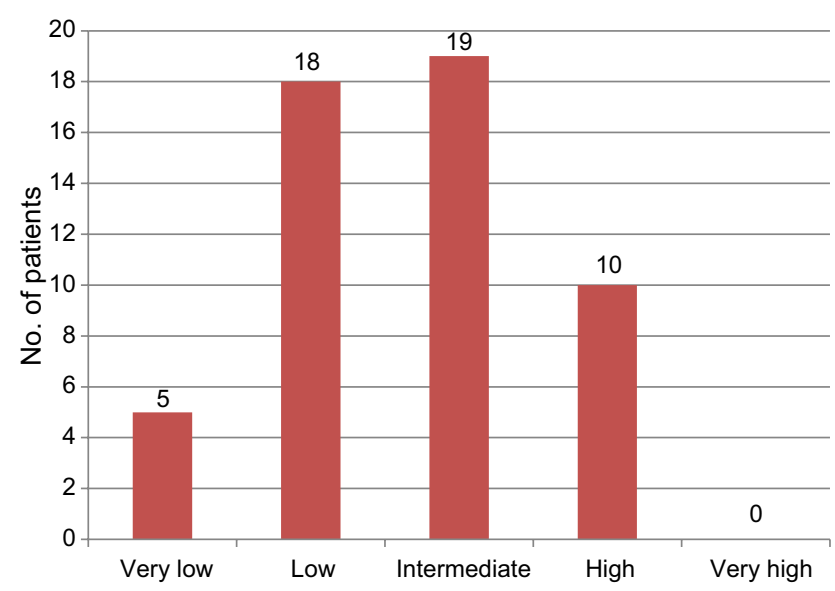

Figure I0 R-IPSS risk stratification of patients.

Abbreviation: R-IPSS, revised international prognostic scoring system.

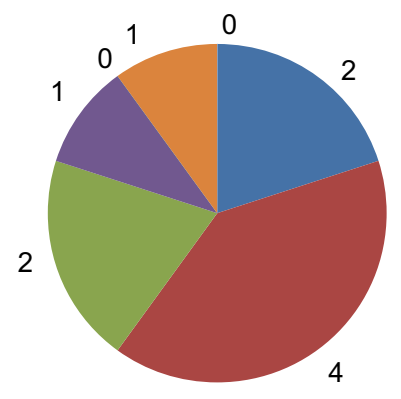

AML MO

AML M1

AML M2

AML M3

AML M4

AML M5

Figure I I Type of AML transformation in patients. Note: Numbers indicate number of patients. Abbreviation: AML, acute myeloid leukemia.

At the end of study period, 40 patients were transfusion dependent for survival. Out of 11 patients with $5 \mathrm{q}$ del, 10 showed good response to lenalidomide and were transfusionfree during the study period. When the factors determining
Total no. of patients

Total no. of patients with leukemic transformation

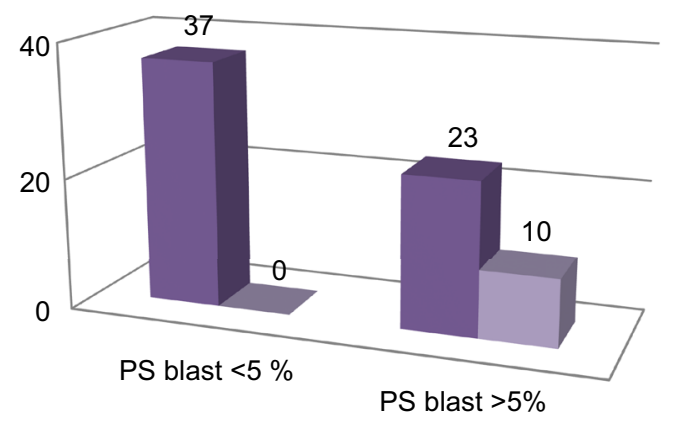

Figure 12 Peripheral smear blast percentage and risk of AML transformation. Abbreviations: AML, acute myeloid leukemia; PS, peripheral smear.

the outcome were studied, it was found that significant association with the outcome was present for prior radiation exposure, percentage of blasts in peripheral smear, and type of MDS. There was significant association between the bone marrow blast $>5 \%$ and bone marrow cytopenia with outcome.

Age, gender, prior exposure to chemotherapeutic agents, hemoglobin, total leucocyte count, and platelet count at the time of diagnosis had no significant bearing with the outcome.

\section{Discussion}

MDSs comprise a heterogeneous group of malignant hematopoietic stem cell disorders characterized by dysplastic and ineffective blood cell production and a variable risk of transformation to AML. In this study, $73 \%$ of patients were aged $>60$ years. Median age was 67 years. These results were concordant with a study published by Ma of US where $86 \%$ of the patients were aged $>60$ years and with another study by Jädersten and Hellström-Lindberg in which majority of the patients $(76 \%)$ were aged $>70$ years. ${ }^{1,2}$ There was no significant association between age and outcome in this study. Furthermore, no association could be obtained between age and transformation to AML. In this study, $70 \%$ of the patients $(n=42)$ were males. In a study by Hofmann and Koeffler, MDS was more common in males. ${ }^{3}$ The reason for male preponderance, as seen in most studies, has not been elucidated. In fact, loss of Y chromosome in cytogenetic analysis of MDS patients renders a good prognosis. In our study, most of the patients were working in agriculture and allied fields, with exposure to pesticides. However, an etiological association could not be established, as ours was not a case-control study. In a French study by Nisse et al, agricultural workers carried a significant risk of developing MDS compared to controls with an odds ratio of 3.66. ${ }^{4}$ Further studies are also needed 
to establish the association between etiological factors and different morphological types of MDSs.

Most common symptom in our study was fatigability followed by dyspnea on exertion and palpitation. Bleeding tendency was a symptom in one-third of the patients. Fever was a less common symptom. Three patients had bone pain, which was attributed to the occurrence of autoimmune phenomena in MDS. Symptoms due to anemia were prominent in most of the studies, whereas symptoms due to leucopenia were less. Bleeding tendency was a frequent symptom in intermediate- and high-risk groups, and was less common in low-risk groups. ${ }^{5}$ In a study by Germing et al, dyspnea on exertion was the commonest symptom. ${ }^{6}$ With fatigability being a nonspecific symptom, most of the patients did not come to seek medical help at an early stage, especially in Indian scenario. Fever was an uncommon symptom in most studies. Other rare symptoms reported in a study by Foran and Shammo include polyuria due to diabetes insipidus and cognitive dysfunction. ${ }^{7}$ In this study, seven patients had past history of other cancers, of which lymphoma was the commonest followed by breast carcinoma. Of these, five patients showed MDS-AML transformation on follow-up. In a study conducted in Tokyo by Ando et al, 13 patients who received treatment for breast cancer developed MDS on follow-up. ${ }^{8}$ Treatment for breast cancer is associated with a significant risk of developing MDS at a later date and was proved in a cohort study by Kaplan etal. ${ }^{9}$ Likewise, risk of MDS developing after treatment for lymphoma, also needs to be studied. In another study by Le Deley et al, there was a positive relationship between the cumulative dose of alkylating agents or topoisomerase II inhibitors and the risk of developing secondary MDS/AML. ${ }^{10}$ In general, the peak incidence of MDS/AML occurs 4-6 years after the initiation of cytotoxic therapy, although latency periods as short as 12 months (in the setting of topoisomerase II inhibitors) and as long as 15-20 years (in the setting of radiation exposure) have been reported.

In this study, there was a significant association between outcome and radiation exposure ( $p$-value $=0.044)$. However, there was no association between radiation exposure and risk of AML transformation. In a Japanese study of atomic bomb survivors conducted by Iwanaga et al, there was a significant correlation between radiation exposure and risk of AML transformation. ${ }^{11}$ Those in the coastal areas where sources of uranium and thorium are high are also at risk of developing MDS. Progression to AML is also accelerated in individuals exposed to radiation.

Most common sign was pallor followed by pedal edema. Though many patients did not have any ophthalmological complaints, retinal hemorrhages were observed in $25 \%$ of the patients. Retinal hemorrhage was an impending sign of intracranial bleeding in many patients. Lymphadenopathy and hepatosplenomegaly, though very uncommon in MDS, were observed in four patients in our study. In a study by Chatterjee et al, organomegaly was observed in many patients, possibly due to inclusion of chronic myelomonocytic leukemia as a type of MDS in their study. ${ }^{12}$

Mean hemoglobin concentration in our study was $5.5 \mathrm{~g} / \mathrm{dL}$. No significant association was found between these parameters and outcome as well as risk of transformation to AML in our study. In the study by Germing et al, low hemoglobin, absolute neutrophil count, and low platelet count were associated with adverse outcome. Hemoglobin concentration was one of the criteria for prognostic assessment in R-IPSS. Thrombocytosis was observed in most of the patients with $5 q$ del MDS. The prognostic significance of thrombocytosis in this subset of patients needs to be studied. In a study by Zikria et al, thrombocytosis in other subsets of MDS was associated with an adverse outcome. ${ }^{13}$

In this study, blast percentage in peripheral smear and cytopenia in bone marrow at the time of diagnosis had a significant association with risk of AML transformation as well as outcome. Patients with initial blast percentage $>5$ had a significant risk of transformation to AML than those with blast percentage $<5$. In a study by Orazi and Czader, cytopenia in bone marrow was associated with an adverse prognostic outcome. ${ }^{14}$

RA was the most common type of MDS in this study. It is to be noted that RARS was less common in our study and in another study conducted by Varma et al. ${ }^{15}$ RARS is much more common in Western population. In our study, RA and RARS patients had better outcome with transfusion-free survival, whereas RCMD patients had severe cytopenia and were transfusion dependent. This is concordant with most of the studies that analyzed the hematopathological types of MDS.

Cytogenetic analysis was performed for 52 out of 60 patients. Of them, $34 \%$ had cytogenetic abnormalities. This is lower compared to a study conducted by Chaubey et al, in which cytogenetic abnormalities were found in $50 \%$ of the patients. ${ }^{16}$ Most common abnormality found in our study was deletion of long arm of fifth chromosome ( $5 \mathrm{q}$ del). Similar finding was observed in a study published by Vundinti et al. ${ }^{17}$ Patients with $5 \mathrm{q}$ deletion were given lenalidomide, and good response was observed after 4 weeks in terms of clinical symptoms and hematological indices. In this study, 10 out of 11 patients with $5 q$ deletion had transfusion-free survival when followed up after 6 months of treatment. 
This observation supports that lenalidomide should be the first choice of drug for patients with $5 \mathrm{q}$ deletion. A longer follow-up study is needed to assess survival rates and therapyrelated outcomes. Use of lenalidomide in the context of other karyotypes needs to be investigated. Next common chromosomal abnormality found was deletion of long arm of seventh chromosome (del 7q). These patients had intermediate prognosis and were transfusion dependent. Patients with loss of Y chromosome had a poor prognosis in our study. Other complex karyotypes were not found.

\section{Conclusion}

This study brings to light the different etiological factors that can predispose to MDS. Radiation exposure, chemotherapeutic agents, and exposure to pesticides pose a major threat. Nonspecific symptoms, difficulty in excluding secondary causes of dysplasia, and heterogeneity of histopathological subtypes often lead to delay in diagnosis. In our study, cytogenetic profiling and application of R-IPSS played a major role in diagnostic and prognostic assessment of patients with MDS. At present, cytogenetic investigations in developing countries are performed in only a few centers. Cytogenetics will add substantially to the understanding of etiopathogenesis and individualization of treatment in MDS. This study supports the superiority of lenalidomide in the treatment of $5 \mathrm{q}$ del in patients with MDS. The impact of lenalidomide in other subsets of MDS needs to be studied.

\section{Disclosure}

The author reports no conflicts of interest in this work.

\section{References}

1. Ma X. Epidemiology of myelodysplastic syndromes. Am J Med. 2012;125(7 Suppl):S2-S5.
2. Jädersten M, Hellström-Lindberg E. Myelodysplastic syndromes: biology and treatment. J Intern Med. 2009;265(3):307-328.

3. Hofmann WK, Koeffler HP. Myelodysplastic syndrome. Annu Rev Med. 2005;56:1-16.

4. Nisse C, Haguenoer JM, Grandbastien B, et al. Occupational and environmental risk factors of the myelodysplastic syndromes in the North of France. Br J Haematol. 2001;112(4):927-935.

5. Bryan J, Jabbour E, Prescott H, Kantarjian H. Thrombocytopenia in patients with myelodysplastic syndromes. Semin Hematol. 2010;47(3): 274-280.

6. Germing U, Kobbe G, Haas R, Gattermann N. Myelodysplastic syndromes: diagnosis, prognosis, and treatment. Dtsc Ärztebl Int. 2013;110(46): 783-790.

7. Foran JM, Shammo JM. Clinical presentation, diagnosis, and prognosis of myelodysplastic syndromes. Am J Med. 2012;125(7 Suppl):S6-S13.

8. Ando M, Narabayashi M, Watanabe T, et al. Therapy-related leukemia and myelodysplastic syndrome in breast cancer patients treated with cyclophosphamide or anthracyclines. Jpn J Clin Oncol. 1999;29(1): 28-32.

9. Kaplan HG, Malmgren JA, Atwood MK. Increased incidence of myelodysplastic syndrome and acute myeloid leukemia following breast cancer treatment with radiation alone or combined with chemotherapy: a registry cohort analysis 1990-2005. BMC Cancer. 2011;11:260.

10. Le Deley MC, Suzan F, Cutuli B, et al. Anthracyclines, mitoxantrone, radiotherapy, and granulocyte colony-stimulating factor: risk factors for leukemia and myelodysplastic syndrome after breast cancer. $J$ Clin Oncol. 2007;25(3):292-300.

11. Iwanaga M, Hsu WL, Soda M, et al. Risk of myelodysplastic syndromes in people exposed to ionizing radiation: a retrospective cohort study of Nagasaki atomic bomb survivors. J Clin Oncol. 2011;29(4):428-434.

12. Chatterjee T, Dixit A, Mohapatra M, et al. Clinical, haematological and histomorphological profile of adult myelodysplastic syndrome. Study of 96 cases in a single institute. Eur J Haematol. 2004;73(2):93-97.

13. Zikria J, Galili N, Tsai WY, Zhan H, Ma X. Thrombocytosis in myelodysplastic syndromes: not an innocent bystander. J Blood Disord Transfus. 2012;S3:002.

14. Orazi A, Czader MB. Myelodysplastic syndromes. Am J Clin Pathol. 2009;132(2):290-305.

15. Varma N, Varma S. Proliferative indices, cytogenetics, immunophenotype and other prognostic parameters in myelodysplastic syndromes. Indian J Pathol Microbiol. 2008;51(1):97-101.

16. Chaubey R, Sazawal S, Dada R, Mahapatra M, Saxena R. Cytogenetic profile of Indian patients with de novo myelodysplastic syndromes. Indian J Med Res. 2011;134(4):452-457.

17. Vundinti BR, Kerketta L, Jijina F, Ghosh K. Cytogenetic study of myelodysplastic syndrome from India. Indian J Med Res. 2009;130(2): $155-159$.
Journal of Blood Medicine

\section{Publish your work in this journal}

The Journal of Blood Medicine is an international, peer-reviewed, open access, online journal publishing laboratory, experimental and clinical aspects of all aspect pertaining to blood based medicine including but not limited to: Transfusion Medicine; Blood collection, Donor issues, Transmittable diseases, and Blood banking logistics; Immunohematology; Artificial and alternative

\section{Dovepress}

blood based therapeutics; Hematology; Biotechnology/nanotechnology of blood related medicine; Legal aspects of blood medicine; Historical perspectives. The manuscript management system is completely online and includes a very quick and fair peer-review system. Visit http://www.dovepress.com/ testimonials.php to read real quotes from published authors. 